\title{
VI. Ventilación no invasiva en pacientes con edema pulmonar agudo cardiogénico
}

\author{
FELIPE ALLER R.* y ORLANDO DÍAZ P.**
}

VI. Noninvasive ventilation in patients with acute cardiogenic pulmonary edema

\begin{abstract}
El propósito de este documento ha sido analizar el estado actual del empleo de la ventilación no invasiva (VNI) en el manejo de la insuficiencia respiratoria aguda que acompaña al edema pulmonar agudo cardiogénico. Con este fin se revisaron los estudios clínicos randomizados (ECR) y las revisiones sistemáticas y meta-análisis publicados hasta ahora, siguiendo las recomendaciones de la Sociedad Americana de Tórax (American Thoracic Society) respecto de la graduación de la calidad de la evidencia para cada situación clínica.
\end{abstract}

\section{Introducción}

El impacto de la insuficiencia cardíaca en la salud pública es muy elevado. En los Estados Unidos, la insuficiencia cardíaca en todas sus formas de presentación constituye la causa más frecuente de hospitalización en personas mayores de 65 años $^{1}$, siendo la estimación de los costos directos e indirectos de 25,8 billones de dólares en $2004^{2}$. Una de sus formas de presentación más grave, el edema pulmonar agudo, motiva más de un millón de consultas anuales y es responsable de la utilización de 6,5 millones días/hospital al año. La mortalidad intrahospitalaria del edema pulmonar agudo (EPA) es alta, oscilando entre $10-20 \%{ }^{3-7}$.

\section{Factores pronósticos}

El edema pulmonar agudo puede presentarse como manifestación de insuficiencia cardíaca aguda, de insuficiencia cardíaca aguda sobre cró- nica, o de insuficiencia cardíaca avanzada o refractaria ${ }^{2}$. Abarca un amplio rango de edades y a menudo se acompaña de otras condiciones cardiovasculares o comorbilidades que pueden precipitarlo directamente o contribuir a ello. Entre las condiciones cardiovasculares se cuentan el infarto del miocardio, arritmias, hipertensión arterial, y defectos valvulares. Las comorbilidades frecuentes son la insuficiencia renal, diabetes mellitus y enfermedades cerebro-vasculares. Aproximadamente la mitad de los pacientes con falla cardíaca aguda posee una función sistólica normal ${ }^{8}$ y un porcentaje similar evoluciona con hipertensión sistólica (presión sistólica mayor de $140 \mathrm{mmHg})^{8,9}$. Asimismo, su gravedad, desde el punto de vista de la disnea y las alteraciones del intercambio gaseoso, es muy diversa, pudiendo o no acompañarse de hipercapnia en ausencia de enfermedad pulmonar ${ }^{10}$. Es, por lo tanto, un cuadro clínico heterogéneo asociado a una mortalidad variable.

Una serie de factores influyen en forma independiente sobre la mortalidad, siendo los más importantes: a) la concomitancia de infarto del miocardio $^{5-7,10}$; b) la edad ${ }^{11}$; c) la presencia de una presión arterial normal (presión sistólica $\leq 140$ $\mathrm{mmHg})^{5,7}$, en contraposición con la hipertensión (presión sistólica $>160 \mathrm{mmHg}$ ) que se asocia a un menor riesgo de muerte ${ }^{9,12}$; d) la necesidad de intubación traqueal ${ }^{6,10}$ y e) algunas comorbilidades. La presencia de disfunción diastólica parece tener pronóstico similar o peor que la disfunción sistólica ${ }^{13,14}$.

El riesgo de intubación fue evaluado en 80 pacientes por Masip y cols ${ }^{10}$, quienes observaron que los factores independientes de riesgo eran infarto del miocardio; acidosis respiratoria

\footnotetext{
* Servicio de Medicina, Instituto Nacional del Tórax.

** Profesor Asociado de Medicina, Departamento de Enfermedades Respiratorias, Pontificia Universidad Católica de Chile.
} 
o mixta $(\mathrm{pH}<7,25)$; hipercapnia $\left(\mathrm{PaCO}_{2}>45\right.$ $\mathrm{mmHg}$ ); y presión sistólica $<140 \mathrm{mmHg}$. Se observó un muy bajo riesgo de intubación en presencia de hipertensión sistólica (mayor de $180 \mathrm{mmHg}$ ), independientemente de la $\mathrm{PaCO}_{2}$ y el $\mathrm{pH}$.

\section{Definiciones}

En el edema pulmonar agudo, comúnmente se ha empleado presión positiva continua en la vía aérea (CPAP) y ventilación con presión de soporte (PSV) asociada a presión positiva al final de la espiración (PEEP). Es conveniente aclarar, sin embargo, que la CPAP no corresponde estrictamente a un modo ventilatorio, pues no aumenta directamente la ventilación. Por tal razón, se empleará el término VNI sólo para referirse a la modalidad de PSV + PEEP. Por otro lado, es frecuente que las publicaciones empleen el término BiPAP ${ }^{\circledR}$ para referirse a la modalidad PSV + PEEP, ya que usualmente se ha utilizado ventiladores portátiles. Sin embargo, tal denominación no es apropiada ya que BiPAP® es la marca registrada por Respironics, Inc. para referirse a dicha modalidad. Comúnmente, cuando se emplea ventiladores portátiles la modalidad PSV + PEEP se denomina binivel, para aclarar que el ventilador ha sido programado para proporcionar una presión inspiratoria (IPAP) y espiratoria (EPAP), en que la presión de soporte inspiratorio corresponde a la resta de IPAP EPAP. Estos aspectos técnicos se aclaran en los capítulos sobre Equipos e Interfaces y Modalidades Ventilatorias.

\section{Fundamentos fisiopatológicos}

La elevación de la presión intratorácica, ya sea con CPAP o VNI, re-expande los alvéolos inundados y recluta aquellos colapsados, lo que se traduce en un aumento de la capacidad residual funcional, mejoría de la distensibilidad del sistema respiratorio y reducción del cortocircuito intrapulmonar. El efecto clínico es un aumento de la $\mathrm{PaO}_{2}$ y reducción de la disnea ${ }^{15,16}$. La CPAP no sólo disminuye sustancialmente el trabajo respiratorio y mejora la oxigenación, sino que puede también tener un efecto benéfico sobre la función cardiovascular. Se ha demostrado que en pacientes con función sistólica preservada, el empleo de CPAP disminuye significativamente el volumen de final de diástole del ventrículo izquierdo, mientras que en aquellos con disfunción sistólica, aumenta además la fracción de eyección ${ }^{16}$. Un efecto similar se ha observado al emplear PSV + PEEP, el que es descrito con mayor detalle en el capítulo Efectos fisiológicos de la VNI.

A diferencia de la VNI, la CPAP no tiene un efecto directo sobre la ventilación alveolar, sino que actúa oponiéndose a los mecanismos fisiopatológicos del edema pulmonar. Consecuentemente, su efecto sobre el trabajo respiratorio y sobre el intercambio gaseoso, específicamente en los pacientes hipercápnicos, puede ser más lento que al aplicar VNI. ${ }^{17}$

\section{Efecto de la VNI en el edema pulmonar agudo cardiogénico}

Preguntas: ¿La ventilación no invasiva reduce la necesidad de ventilación mecánica (VM) invasiva y la estadía hospitalaria? ¿La VNI reduce el riesgo de complicaciones y muerte en pacientes con edema pulmonar agudo?

Pacientes: Pacientes con edema pulmonar agudo cardiogénico en insuficiencia respiratoria.

Intervención: VNI en pacientes con edema pulmonar agudo cardiogénico.

Objetivo: Evaluar si la VNI reduce la necesidad de intubación endotraqueal, la estadía hospitalaria y la mortalidad en pacientes con edema pulmonar cardiogénico.

\section{Resumen de la evidencia}

Estudios clínicos randomizados (ECR). Dos décadas atrás, Räsänen y $\operatorname{cols}^{18}$, demostraron que el uso de CPAP por 3 horas en pacientes con EPA producía una mejoría más rápida del intercambio gaseoso y una mayor reducción del trabajo respiratorio que el tratamiento estándar. Sin embargo, no lograron demostrar un efecto estadísticamente significativo sobre la tasa de intubación endotraqueal ni sobre la mortalidad. Numerosos ECR han sido publicados desde entonces, con resultados discordantes (Tablas 1$3)^{17,19-39}$. El último de ellos, aparecido durante la preparación del presente documento, será discutido en detalle al final ${ }^{4}$.

Los ECR mencionados han incluido un número muy variable de enfermos, que en los grupos tratados ha oscilado entre $9^{26}$ y $65^{34}$, sin mencionar los 702 del estudio más reciente ${ }^{4}$. En los grupos control, la tasa de intubación ha variado entre $0^{29}$ y $60 \%{ }^{18}$; la mortalidad general entre $0^{26}$ y $64 \%{ }^{22}$; y la tasa de nuevos infartos del miocardio (esto es, aquellos ocurridos después del ingreso al hospital) entre $0^{35}$ y $100 \%{ }^{22}$. 
Tabla 1. Resumen de los estudios clínicos randomizados que han comparado presión positiva continua en la vía aérea (CPAP) con terapia estándar

\begin{tabular}{lcccc}
\hline Primer autor & Año & $\begin{array}{c}\text { Lugar } \\
\text { aplicación }\end{array}$ & $\begin{array}{c}\text { Mortalidad } \\
\text { Estándar/CPAP* }\end{array}$ & $\begin{array}{c}\text { Intubación } \\
\text { Estándar/CPAP } \dagger\end{array}$ \\
\hline Räsänen $^{18}$ & 1985 & nd & $6(14) / 3(17)$ & $12(8) / 6(14)$ \\
Bersten $^{19}$ & 1991 & DE/UCI & $4(16) / 2(17)$ & $7(13) / 0(19)$ \\
Lin $^{20}$ & 1995 & DE & $6(44) / 4(46)$ & $18(32) / 8(42)$ \\
Takeda $^{21}$ & 1997 & UCI & $3(12) / 1(14)$ & $6(9) / 1(14)$ \\
Takeda $^{22}$ & 1998 & UC & $7(4) / 1(10)$ & $8(3) / 2(9)$ \\
Delclaux $^{23}$ & 2000 & nd & $7(13) / 7(15)$ & $6(14) / 6(16)$ \\
Park $^{26}$ & 2001 & nd & $0(10) / 1(8)$ & $4(6) / 3(6)$ \\
Kelly $^{29}$ & 2002 & DE & $7(24) / 2(25)$ & $0(31) / 0(27)$ \\
Hao $^{28}$ & 2002 & nd & nd & $9(17) / 1(24)$ \\
Crane $^{36}$ & 2004 & DE & $6(14) / 0(20)$ & $0(20) / 1(19)$ \\
L'Her $^{37}$ & 2004 & DE & $14(32) / 12(31)$ & $4(42) / 2(41)$ \\
Park $^{38}$ & 2004 & DE & $6(20) / 1(26)$ & $11(15) / 2(25)$ \\
\hline
\end{tabular}

DE: Departamento de emergencia; UCI: Unidad de cuidados intensivos; UC: Unidad coronaria; nd: no disponible. *Los datos representan pacientes que mueren (sobreviven). $\dagger$ Los datos indican los pacientes que se intuban (no se intuban).

Tabla 2. Resumen de los estudios clínicos randomizados que han comparado ventilación no invasiva (VNI) con terapia estándar

\begin{tabular}{|c|c|c|c|c|}
\hline Primer autor & Año & $\begin{array}{c}\text { Lugar } \\
\text { aplicación }\end{array}$ & $\begin{array}{c}\text { Mortalidad } \\
\text { Estándar/VNI* }\end{array}$ & $\begin{array}{c}\text { Intubación } \\
\text { Estándar/VNI } †\end{array}$ \\
\hline Masip $^{24}$ & 2000 & DE/UCI & $2(16) / 0(19)$ & $6(12) / 1(18)$ \\
\hline Levitt $^{25}$ & 2001 & DE & $3(14) / 3(18)$ & $7(10) / 5(16)$ \\
\hline Park $^{26}$ & 2001 & nd & $0(10) / 0 \quad(7)$ & $\begin{array}{llll}4 & (6) / & 0 & \text { (7) }\end{array}$ \\
\hline $\mathrm{Nava}^{34}$ & 2003 & $\mathrm{DE}$ & $9(56) / 6(59)$ & $16(49) / 13(52)$ \\
\hline Ferrer $^{32}$ & 2003 & UCI & $2(13) / 1(14)$ & $2(13) / 1(14)$ \\
\hline Crane $^{36}$ & 2004 & $\mathrm{DE}$ & $6(14) / 5(15)$ & $0(20) / 1(19)$ \\
\hline Park $^{38}$ & 2004 & $\mathrm{DE}$ & $6(20) / 2(25)$ & $11(15) / 2(25)$ \\
\hline
\end{tabular}

DE: Departamento de emergencia; UCI: Unidad de cuidados intensivos; UC: Unidad coronaria; nd: no disponible. *Los datos representan pacientes que mueren (sobreviven). $\dagger$ Los datos indican los pacientes que se intuban (no se intuban).

Tabla 3. Resumen de los estudios clínicos randomizados que han comparado CPAP con VNI

\begin{tabular}{lcccc}
\hline Primer autor & Año & $\begin{array}{c}\text { Lugar } \\
\text { aplicación }\end{array}$ & $\begin{array}{c}\text { Mortalidad } \\
\text { CPAP/VNI* }\end{array}$ & $\begin{array}{c}\text { Intubación } \\
\text { CPAP/VNI } \dagger\end{array}$ \\
\hline Metha $^{17}$ & 1997 & DE & $2(11) / 1(13)$ & $1(12) / 1(13)$ \\
Park $^{26}$ & 2001 & nd & $1(8) / 0(7)$ & $3(6) / 0(7)$ \\
Bollaert $^{27}$ & 2002 & nd & $4(15) / 4(13)$ & $4(15) / 5(12)$ \\
Martin-Bermúdez $^{30}$ & 2002 & nd & $5(34) / 2(39)$ & nd \\
Liesching $^{33}$ & 2003 & nd & nd & $4(32) / 1(34)$ \\
Cross $^{31}$ & 2003 & DE & $5(31) / 3(32)$ & $1(19) / 1(19)$ \\
Crane $^{36}$ & 2004 & DE & $0(20) / 5(15)$ & $1(21) / 2(22)$ \\
Bellone $^{35}$ & 2004 & DE & $2(20) / 0(24)$ & $2(25) / 2(25)$ \\
Park $^{38}$ & 2004 & DE & $1(26) / 2(25)$ & $1(17) / 2(16)$ \\
Bellone $^{39}$ & 2005 & DE & $1(17) / 0(18)$ & $(13)$ \\
\hline
\end{tabular}

DE: Departamento de emergencia; nd: no disponible. *Los datos representan pacientes que mueren (sobreviven). $\nmid$ Los datos indican los pacientes que se intuban (no se intuban). 
Estos datos indican que existe una notoria heterogeneidad en la gravedad de los pacientes reclutados en los diferentes estudios. Como se observa en las Tablas 1-3, el lugar de aplicación de la VNI también ha sido variable, pues se ha realizado en ambientes protegidos de UCI o unidades coronarias, pero no menos frecuentemente en salas de emergencia. Aunque los criterios para decidir la intubación fueron explícitos en la mayoría de los estudios, han sido "subjetivos" en otros; es decir, dependieron del criterio de los médicos tratantes. Es interesante notar que en aquellos ECR en que el criterio de intubación no fue explícito, las tasas de intubación fueron notoriamente bajas ${ }^{4,17,29,38}$, lo que sugiere que esta variable de desenlace puede ser influida por criterios a priori demasiado estrictos. Así, por ejemplo, Crane y cols ${ }^{36}$, observaron que seis pacientes cumplieron con sus criterios de intubación, pero sólo dos fueron finalmente intubados; los cuatro restantes evolucionaron sin complicaciones con terapia médica usual.

Mientras que el empleo de CPAP se considera un procedimiento seguro, no ha sucedido lo mismo con la PSV + PEEP. Esta duda surgió del estudio de Mehta y cols ${ }^{17}$, quienes observaron una alta frecuencia de nuevos infartos del miocardio con el empleo de PSV + PEEP, lo que obligó a la detención prematura de su ensayo clínico. Sus resultados no fueron concluyentes debido al número reducido de pacientes reclutados, pero una serie de ECR subsecuentes no confirmó este hallazgo $0^{4,24,34-36,38}$. Se ha sugerido, en consecuencia, que la mayor tasa de infarto del miocardio comunicada por Metha y cols. en los pacientes tratados con PSV + PEEP probablemente se debió a que un mayor número de ellos tenía un infarto en evolución al momento de incorporarse al estudio.

Los equipos empleados para proporcionar CPAP o VNI han variado entre los distintos ECR. La modalidad de CPAP se ha aplicado con equipos ad-hoc de alto flujo, con ventiladores convencionales y con ventiladores portátiles. Similar variabilidad se observa en los equipos empleados para proporcionar PSV + PEEP, ya que se han empleado ventiladores convencionales y portátiles. Como los ventiladores convencionales poseen una amplia capacidad de monitorización y la posibilidad de administrar controladamente el oxígeno, Masip y cols, han sugerido que la selección del equipo puede influir en los resultados, particularmente cuando se emplea PSV + PEEP ${ }^{40}$.

Los parámetros empleados con CPAP y PSV + PEEP no son menos variables. Por ejemplo, en aquellos estudios que han comparado CPAP y PSV + PEEP se ha usado CPAP fija (10 $\left.\mathrm{cmH}_{2} \mathrm{O}\right)^{17,35,36,39}$, o ajustada de acuerdo a las "necesidades clínicas" $\left(5-20 \mathrm{cmH}_{2} \mathrm{O}\right)^{26,31,38}$, al igual que la PSV + PEEP, que ha sido fija en algunos (15 y $5 \mathrm{cmH}_{2} \mathrm{O}$, respectivamente) $)^{17,36}$ o ajustada de acuerdo a los requerimientos clínicos ${ }^{26,31,35,38,39}$. Cuando estos "requerimientos clínicos" han sido explícitos han tenido como objetivo lograr la menor $\mathrm{FIO}_{2}$ posible y/o a reducir la disnea.

Revisiones sistemáticas y meta-análisis. Se han publicado nueve revisiones sistemáticas y/o meta-análisis ${ }^{41-49}$. La primera revisión sistemática, publicada en 1998 por Pang y cols ${ }^{46}$, no logró demostrar una reducción significativa de la tasa de intubación ni de la mortalidad al emplear CPAP, pero sólo incluyó tres estudios $(\mathrm{n}=179)$. Las revisiones sistemáticas y metaanálisis posteriores, que han incluido un número sustancialmente mayor de pacientes, fueron publicados entre 2005-2006, y se diferencian básicamente por el número de ECR incluidos. De ellos, el meta-análisis de Peter y $\operatorname{cols}^{47}$, es el más numeroso, está razonablemente bien diseñado y los criterios de selección, métodos de extracción de datos y análisis, claramente descritos. Incorporó 23 estudios en que se comparó CPAP con terapia estándar (12 estudios; $\mathrm{n}=$ 583), VNI con terapia estándar (7 estudios; $\mathrm{n}=$ 345), y CPAP con VNI (10 estudios; $n=406$ ). Nótese que tres estudios fueron incluidos en las 3 categorías, ya que contaban con tres ramas (terapia estándar, CPAP y VNI) ${ }^{26,36,38}$. El empleo de CPAP disminuyó significativamente la mortalidad hospitalaria de 25 a $13 \%$ al compararse con la terapia usual (riesgo relativo [RR] 0,59; intervalo de confianza [IC] 95\%: 0,38 a 0,90 ; $\mathrm{p}=0,015)$, efecto que no se demostró con VNI. Tanto la CPAP como la VNI redujeron significativamente la tasa de intubación en comparación con el tratamiento estándar, de 29 a $11 \%$ (RR 0,44; IC 95\%: 0,29 a 0,66; p = 0,0003) y de 27 a 13\% (RR 0,50; IC 95\%: 0,27 a 0,90; $\mathrm{p}=0,02)$, respectivamente. Ninguna de las técnicas redujo la estadía hospitalaria. La tasa de nuevos eventos coronarios fue ligeramente superior con el empleo de VNI que con CPAP (RR 1,49; IC 95\%: 0,92 a 2,42; p = 0,11). Los autores hacen notar que la potencia del metaanálisis es sólo moderada para comparar los efectos de CPAP vs terapia estándar e insuficiente para VNI $v s$ terapia estándar y CPAP $v s$ VNI, al menos en términos de la mortalidad. No se encontró un efecto sustancial del riesgo subyacente sobre la mortalidad ni la tasa de intubación al comparar CPAP o VNI con la terapia estándar. 
Los resultados de este meta-análisis son muy similares a los obtenidos por Wynck y cols ${ }^{49}$, lo que no es de extrañar ya que este último incluyó 17 de los 23 estudios utilizados por Peter y cols en sus análisis.

Estudio multicéntrico 3CPO. El estudio 3CPO (Three Interventions in Cardiogenic Pulmonary Oedema) fue realizado en 23 salas de emergencia del Reino Unido y reclutó 1.069 pacientes con edema pulmonar agudo y $\mathrm{pH}$ arterial menor de 7,35 en el transcurso de cuatro años (2003-2007). Los pacientes fueron randomizados a oxígeno suplementario, CPAP o VNI, recibiendo todos similar terapia estándar. En los tres grupos se aplicó oxígeno suficiente para mantener una $\mathrm{SaO}_{2}$ mayor de 92\%. La CPAP se inició a $5 \mathrm{cmH}_{2} \mathrm{O}$, pudiendo aumentarse hasta 15 $\mathrm{cmH}_{2} \mathrm{O}$. La VNI se inició a una IPAP de 8 $\mathrm{cmH}_{2} \mathrm{O}$ y una PEEP de $4 \mathrm{cmH}_{2} \mathrm{O}$, pudiendo incrementarse hasta 20 y $10 \mathrm{cmH}_{2} \mathrm{O}$, respectivamente. Los tratamientos asignados se mantuvieron por un mínimo de 2 horas. La necesidad de continuar estas terapias después de este período quedó a discreción del médico tratante, al igual que la decisión de intubación. Se evaluó gases arteriales y disnea al cabo de 1 y 2 horas. El desenlace primario fue la mortalidad o intubación a 7 días. La edad promedio de los pacientes fue 78 años, alrededor de un $20 \%$ tenía síntomas de infarto del miocardio, la presión sistólica promedio fue de $161 \mathrm{mmHg}$ y la $\mathrm{PaCO}_{2}$ de aproximadamente $56 \mathrm{mmHg}$. A los 7 días no hubo diferencias en la mortalidad entre las tres ramas $(9,8 \%$ en los controles y $9,5 \%$ en el grupo CPAP/VNI). Diez de los 367 controles y 20 de los 702 pacientes tratados con CPAP/ VNI fueron intubados. De esta manera, el desenlace combinado mortalidad o intubación a 7 días, al igual que la estadía en el hospital, tampoco fueron significativamente diferentes entre los tres grupos. A su vez, la mortalidad a 30 días fue también similar. El único efecto significativo de la rama de CPAP/VNI comparado con oxigenoterapia fue una reducción más rápida de la disnea, frecuencia cardíaca e hipercapnia. Un número mayor de pacientes discontinuó la VNI o CPAP que el oxígeno, debido a mala tolerancia. Por el contrario, la oxigenoterapia fue discontinuada más frecuentemente que CPAP o VNI por empeoramiento de los gases arteriales o disnea. El número de pacientes que desarrolló un infarto del miocardio dentro de la hospitalización fue similar entre los tres grupos. En los análisis post-hoc no se observó una interacción entre el efecto del tratamiento y la gravedad del
EPA definida por el $\mathrm{pH}$ basal, y la concomitancia de infarto del miocardio, aunque hubo una tendencia con la presión sistólica $(\mathrm{p}=0,17)$. No se apreció interacción entre el efecto del tratamiento y otros factores que podrían influir en el pronóstico del EPA, como la edad, el género y la historia previa de insuficiencia cardíaca.

El estudio 3CPO supera en número al metaanálisis de Peter y $\operatorname{cols}^{47}$, y contradice sus resultados, algo que ha sido previamente comunicado en otras áreas ${ }^{50}$. Sus resultados son probablemente aplicables a una alta proporción de pacientes con EPA, ya que la tasa de reclutamiento fue de $62 \%$ en el grupo asignado a tratamiento, a diferencia de las bajas tasas de estudios previos (entre 10-30\%) ) $^{47}$. Independientemente de ello, aporta otros datos de interés, como cifras de mortalidad razonables, ya que son similares ${ }^{11}$ o sólo ligeramente más elevadas que estudios observacionales recientes ${ }^{51,52}$, y una tasa de intubación notablemente menor que muchos ECR previos. Esto último podría explicarse porque al dejar esta decisión a los médicos tratantes, los criterios para decidir la conexión a ventilación mecánica fueron probablemente más liberales. Aunque el estudio incluye una población principalmente hipercápnica, este grupo es el que aparentemente se beneficia más de $\mathrm{VNI}^{34}$. Por otro lado, cabe comentar que si bien CPAP/VNI aceleran la mejoría de la disnea y el intercambio de gases, estos efectos difícilmente justifican su empleo rutinario en estos pacientes. Sin embargo, debe también resaltarse que CPAP/VNI no se asociaron a efectos adversos, por lo que la utilización de estas técnicas en pacientes que no han respondido a la terapia médica estándar inicial podría aún justificarse.

\section{Efecto potencial}

Debido a la heterogeneidad de los ECR empleados en meta-análisis y a la consistencia de los hallazgos de un gran estudio multicéntrico más reciente, la evidencia indica que el uso rutinario de CPAP o VNI no reduce la mortalidad, la estadía intrahospitalaria ni la necesidad de intubación endotraqueal en pacientes con edema pulmonar agudo cardiogénico. Sólo aceleraría la mejoría de la disnea y del intercambio pulmonar de gases. Sin embargo, no es posible descartar la posibilidad que estas técnicas sean de utilidad en pacientes en los que la terapia estándar ha fracasado.

\section{Evaluación del costo/beneficio}

De acuerdo a la información disponible, el empleo rutinario de CPAP o VNI en estos pacientes no resulta costo/efectivo. 


\section{Grado de recomendación}

El presente consenso no recomienda el empleo rutinario de CPAP o VNI en pacientes con edema pulmonar agudo cardiogénico, ya que no reduce la mortalidad, la estadía intrahospitalaria ni la tasa de intubación (recomendación fuerte sustentada en evidencia de moderada calidad).

\section{Bibliografía}

1.- THOM T, HAASE N, ROSAMOND W, HOWARD VJ, RUMSFELD J, MANOLIO T, et al. Heart Disease and Stroke Statistics-2006 Update: A report from the American Heart Association Statistics Committee and Stroke Statistics Subcommittee. Circulation 2006; 113 : e85-151.

2.- GHEORGHIADE M, ZANNAD F, SOPKO G, KLEIN L, PINA I L, KONSTAM M A, et al, for the International Working Group on Acute Heart Failure Syndromes. Acute heart failure syndromes: Current state and framework for future research. Circulation 2005; 112: 3958-68.

3.- GIROU E, BRUN-BUISSON C, TAILLE S, LEMAIRE F, BROCHARD L. Secular trends in nosocomial infections and mortality associated with noninvasive ventilation in patients with exacerbation of COPD and pulmonary edema. JAMA 2003; 290: 2985-91.

4.- GRAY A, GOODACRE S, NEWBY D E, MASSON M, SAMPSON F, NICHOLL J, the CPO Trialists. Noninvasive ventilation in acute cardiogenic pulmonary edema. N Engl J Med 2008; 359: 142-51.

5.- FIUTOWSKI M, WASZYROWSKI T, KRZEMIDSKAPAKULA M, KASPRZAK J. Pulmonary edema prognostic score predicts in-hospital mortality risk in patients with acute cardiogenic pulmonary edema. Heart Lung 2008; 37: 46-53.

6.- EDOUTE Y, ROGUIN A, BEHAR D, REISNER S. Prospective evaluation of pulmonary edema. Crit Care Med 2000; 28: 330-5.

7.- ADNET F, LE TOUMELIN P, LEBERRE A, MINADEO J, LAPOSTOLLE F, PLAISANCE P, et al. In-hospital and long-term prognosis of elderly patients requiring endotracheal intubation for lifethreatening presentation of cardiogenic pulmonary edema. Crit Care Med 2001; 29: 891-5.

8.- GANDHI S K, POWERS J C, NOMEIR A-M, FOWLE $\mathrm{K}$, KITZMAN D W, RANKIN $\mathrm{K} \mathrm{M}$, et al. The pathogenesis of acute pulmonary edema associated with hypertension. N Engl J Med 2001; 344: 17-22.

9.- GHEORGHIADE M, ABRAHAM W T, ALBERT N M, GREENBERG B H, O'CONNOR C M, SHE L, et al. Systolic blood pressure at admission, clinical characteristics, and outcomes in patients hospitalized with acute heart failure. JAMA 2006; 296: 2217-26.

10.- MASIP J, PÁEZ J, MERINO M, PAREJO S, VECILLA F, RIERA C, et al. Risk factors for intubation as a guide for noninvasive ventilation in patients with severe acute cardiogenic pulmonary edema. Intensive Care Medicine 2003; 29: 1921-8.

11.- LEE D S, AUSTIN P C, ROULEAU J L, LIU P P, NAIMARK D, TU J V. Predicting mortality among patients hospitalized for heart failure: Derivation and validation of a clinical model. JAMA 2003; 290: 25817.

12.- GHEORGHIADE M, FONAROW G C. Systolic blood pressure and outcomes in patients hospitalized with acute heart failure-Reply. JAMA 2007; 297: 808-9.

13.- VASAN R S, LARSON M G, BENJAMIN E J, EVANS J C, REISS C K, LEVY D. Congestive heart failure in subjects with normal versus reduced left ventricular ejection fraction: Prevalence and mortality in a population-based cohort. J Am Coll Cardiol 1999; 33: 1948-55.

14.- TRIBOUILLOY C, RUSINARU D, MAHJOUB H, SOULIERE V, LEVY F, PELTIER M, et al. Prognosis of heart failure with preserved ejection fraction: a 5 year prospective population-based study. Eur Heart J. 2008; 29: 339-47.

15.- LENIQUE F, HABIS M, LOFASO F, DUBOIS-RANDE J L, HARF A, BROCHARD L. Ventilatory and hemodynamic effects of continuous positive airway pressure in left heart failure. Am J Respir Crit Care Med 1997; 155: 500-5.

16.- BENDJELID K, SCHUTZ N, SUTER P M, FOURNIER G, JACQUES D, FAREH S, et al. Does continuous positive airway pressure by face mask improve patients with acute cardiogenic pulmonary edema due to left ventricular diastolic dysfunction? Chest 2005; 127: 1053-8.

17.- MEHTA S, JAY G, WOOLARD R H, HIPONA R A, CONNOLLY E M, CIMINI D M, et al. Randomized, prospective trial of bilevel versus continuous positive airway pressure in acute pulmonary edema. Crit Care Med 1997; 25: 620-8.

18.- RÄSÄNEN J, HEIKKILÄ J, DOWNS J, NIKKI P, VÄISÄNEN I, VIITANEN A. Continuous positive airway pressure by face mask in acute cardiogenic pulmonary edema. Am J Cardiol 1985; 55: 296-300.

19.- BERSTEN A D, HOLT A W, VEDIG A E, SKOWRONSKI G A, BAGGOLEY C J. Treatment of severe cardiogenic pulmonary edema with continuous positive airway pressure delivered by face mask. $\mathrm{N}$ Engl J Med 1991; 325: 1825-30.

20.- LIN M, YANG Y-F, CHIANG H-T, CHANG M-S, CHIANG B N, CHEITLIN M D. Reappraisal of continuous positive airway pressure therapy in acute cardiogenic pulmonary edema: Short-term results and long-term follow-up. Chest 1995; 107: 1379-86.

21.- TAKEDA S, TAKANO T, OGAWA R. The effect of nasal continuous positive airway pressure on plasma endothelin-1 concentrations in patients with severe cardiogenic pulmonary edema. Anesth Analg 1997; 84: 1091-6.

22.- TAKEDA S, NEJIMA J, TAKANO T, NAKANISHI K, TAKAYAMA M, SAKAMOTO A, et al. Effect of nasal continuous positive airway pressure on pulmonary edema complicating acute myocardial infarction. Jpn Circ J 1998; 62: 553-8.

23.- DELCLAUX C, L'HER E, ALBERTI C, MANCEBO J, ABROUG F, CONTI G, et al. Treatment of acute hypoxemic nonhypercapnic respiratory insufficiency with continuous positive airway pressure delivered by a face mask: A randomized controlled trial. JAMA. 2000; 284: 2352-60.

24.- MASIP J, BETBESÉ A, PÁEZ J, VECILlA F, CAÑIZARES R, PADRÓ $J$, et al. Non-invasive 
pressure support ventilation versus conventional oxygen therapy in acute cardiogenic pulmonary oedema: a randomised trial. Lancet 2000; 356: 2126-32.

25.- LEVITT M A. A prospective, randomized trial of BiPAP in severe acute congestive heart failure. J Emerg Med 2001; 21: 363-9.

26.- PARK M, LORENZI-FILHO G, FELTRIM M, VIECILI P, SANGEAN M, VOLPE M, et al. Oxygen therapy, continuous positive airway pressure, or noninvasive bilevel positive pressure ventilation in the treatment of acute cardiogenic pulmonary edema. Arq Bras Cardiol 2001; 76: 221-30.

27.- RUSTERHOLTZ T, BOLLAERT P, FEISSEL M, ROMANO-GIRARD F, HARLAY M L, ZAEHRINGER $\mathrm{M}$, et al. Continuous positive airway pressure $v s$ proportional assist ventilation for noninvasive ventilation in cardiogenic pulmonary edema. Intensive Care Med 2008; 34: 840-6.

28.- HAO C, LUO X, LIU Y. Treatment of severe cardiogenic pulmonary edema with continuous positive airway pressure by nasal face mask. Acta Academiae Medicinae Jiangxi 2002; 42: 48-50.

29.- KELLY C A, NEWBY D E, MCDONAGH T A, MACKAY $\mathrm{T}$ W, BARR J, BOON $\mathrm{N}$ A, et al. Randomised controlled trial of continuous positive airway pressure and standard oxygen therapy in acute pulmonary oedema. Effects on plasma brain natriuretic peptide concentrations. Eur Heart J 2002; 23: 1379 86.

30.- MARTIN-BERMUDEZ R, RODRÍGUEZ-PORTAL J, GARCÍA-GARMENDIA J. Non-invasive ventilation in cardiogenic pulmonay edema. Preliminary results of a randomized trial. Intensive Care Med 2002; 28: A255.

31.- CROSS A M, CAMERON P, KIERCE M, RAGG M, KELLY A M. Non-invasive ventilation in acute respiratory failure: a randomised comparison of continuous positive airway pressure and bi-level positive airway pressure. Emerg Med J 2003; 20: 531-4.

32.- FERRER M, ESQUINAS A, LEÓN M, GONZÁLEZ G, ALARCÓN A, TORRES A. Noninvasive ventilation in severe hypoxemic respiratory failure: A randomized clinical trial. Am J Respir Crit Care Med 2003; 168: 1438-44.

33.- LIESCHING T, CROMIER K, NELSON D. Bilevel noninvasive ventilation versus continuous positive airway pressure to treat acute pulmonary edema. Am J Respir Crit Care Med 2003; 167: A864.

34.- NAVA S, CARBONE G, DIBATTISTA N, BELLONE A, BAIARDI P, COSENTINI R, et al. Noninvasive ventilation in cardiogenic pulmonary edema: A multicenter randomized trial. Am J Respir Crit Care Med 2003; 168: 1432-7.

35.- BELLONE A, MONARI A, CORTELLARO F, VETTORELLO M, ARLATI S, COEN D. Myocardial infarction rate in acute pulmonary edema: Noninvasive pressure support ventilation versus continuous positive airway pressure. Crit Care Med 2004; 32: 1860-5.

36.- CRANE S D, ELLIOTT M W, GILLIGAN P, RICHARDS K, GRAY A J. Randomised controlled comparison of continuous positive airways pressure, bilevel non-invasive ventilation, and standard treatment in emergency department patients with acute cardiogenic pulmonary oedema. Emerg Med J. 2004; 21: 155-61.
37.- L'HER E, DUQUESNE F, GIROU E, ROSIERE X, CONTE P, RENAULT $S$, et al. Noninvasive continuous positive airway pressure in elderly cardiogenic pulmonary edema patients. Intensive Care Medicine. 2004; 30: 882-8.

38.- PARK M, SANGEAN M, VOLPE M D S, FELTRIM M I Z, NOZAWA E, LEITE P F, et al. Randomized, prospective trial of oxygen, continuous positive airway pressure, and bilevel positive airway pressure by face mask in acute cardiogenic pulmonary edema. Crit Care Med 2004; 32: 2407-15.

39.- BELLONE A, VETTORELLO M, MONARI A, CORTELLARO F, COEN D. Noninvasive pressure support ventilation $v s$ continuous positive airway pressure in acute hypercapnic pulmonary edema. Intensive Care Medicine 2005; 31: 807-11.

40.- MASIP J, PÁEZ J, BETBESE A J, VECILLA F, NAVA $S$. Noninvasive ventilation for pulmonary edema in the emergency room. Am J Respir Crit Care Med 2004; 169: 1072-3.

41.- AGARWAL R, AGGARWAL A N, GUPTA D, JINDAL $\mathrm{S}$ K. Non-invasive ventilation in acute cardiogenic pulmonary oedema. Postgrad Med J 2005; 81: 63743.

42.- COLLINS S, MIELNICZUK L, WHITTINGHAM H, BOSELEY M, SCHRAMM D, STORROW A. The use of noninvasive ventilation in emergency department patients with acute cardiogenic pulmonary edema: a systematic review. Ann Emerg Med 2006; 48: 260-9.

43.- HO K, WONG K. A comparison of continuous and bilevel positive airway pressure non-invasive ventilation in patients with acute cardiogenic pulmonary oedema: a meta-analysis. Critical Care 2006; 10: R49.

44.- MASIP J, ROQUE M, SANCHEZ B, FERNANDEZ R, SUBIRANA M, EXPOSITO J A. Noninvasive ventilation in acute cardiogenic pulmonary edema: Systematic review and meta-analysis. JAMA 2005; 294: 3124 30.

45.- NADAR S, PRASAD N, TAYLOR R S, LIP G Y H. Positive pressure ventilation in the management of acute and chronic cardiac failure: a systematic review and meta-analysis. International Journal of Cardiology 2005; 99: 171-85.

46.- PANG D, KEENAN S P, COOK D J, SIBBALD W J. The effect of positive pressure airway support on mortality and the need for intubation in cardiogenic pulmonary edema: A systematic review. Chest 1998; 114: 1185-92.

47.- PETER J V, MORAN J L, PHILLIPS-HUGHES J, GRAHAM P, BERSTEN A D. Effect of non-invasive positive pressure ventilation (NIPPV) on mortality in patients with acute cardiogenic pulmonary oedema: a meta-analysis. Lancet 2006; 367: 1155-63.

48.- SEAN P C, LISA M M, HEATHER A W, MARK E B, DAVID R S, ALAN B S. The use of noninvasive ventilation in emergency department patients with acute cardiogenic pulmonary edema: A systematic review. Annals of Emergency Medicine 2006; 48: 2609.e264.

49.- WINCK J, AZEVEDO L, COSTA-PEREIRA A, ANTONELLI M, WYATT J. Efficacy and safety of non-invasive ventilation in the treatment of acute cardiogenic pulmonary edema - a systematic review and meta-analysis. Critical Care 2006; 10: R69. 
50.- LELORIER J, GREGOIRE G, BENHADDAD A, LAPIERRE J, DERDERIAN F. Discrepancies between meta-analyses and subsequent large randomized, controlled trials. N Engl J Med 1997; 337: 536-42.

51.- NIEMINEN M S, BRUTSAERT D, DICKSTEIN K, DREXLER H, FOLLATH F, HARJOLA V-P, et al, on behalf of the EuroHeart Survey Investigators. EuroHeart Failure Survey II (EHFS II): a survey on hospitalized acute heart failure patients: description of population. Eur Heart J 2006; 27: 2725-36.

52.- ADAMS K J, FONAROW G, EMERMAN C, LEJEMTEL T, COSTANZO M, ABRAHAM W, et al. ASACa. Characteristics and outcomes of patients hospitalized for heart failure in the United States: rationale, design, and preliminary observations from the first 100,000 cases in the Acute Decompensated Heart Failure National Registry (ADHERE). Am Heart J 2005; 149: 209-16.

Correspondencia a:

Dr. Felipe Aller R.

E-mail: faller@manquehue.net 